\title{
Practicing self-compassion weakens the relationship between fear of receiving compassion and the desire to conceal negative experiences from others
}

\author{
Jessica R. Dupasquier, Allison C. Kelly, David A. Moscovitch, and Vanja Vidovic
} Centre for Mental Health Research, University of Waterloo, Waterloo, Canada

\begin{abstract}
Author Note
Funding: This research was supported by funding from the Social Sciences and Humanities Research Council (first, second, and third authors) and the Canada Research Chairs Program (third author).

Conflicts of interest: The authors declare they have no conflict of interest.

Acknowledgments: Special thanks to Ariella Lenton-Brym for her assistance organizing and collecting the data for this project.

This is a post-peer-review, pre-copyedit version of an article published in Mindfulness. The final authenticated version is available online from Springer at: http://dx.doi.org/10.1007/s12671-0170792-0
\end{abstract}




\begin{abstract}
Disclosure of personal distress is linked to important inter- and intrapersonal benefits. However, people who tend to view self-disclosure as being risky are likely to conceal their feelings and forgo opportunities to receive valuable social support. One such group of people may be those who fear receiving compassion. The current study of 85 female undergraduates investigated (a) whether fear of receiving compassion would predict decreased distress disclosure, and (b) whether inducing a self-compassionate mindset could help to temper the association between fear of receiving compassion and perceived risks of revealing one's distress to others. Participants completed self-report questionnaires to measure trait-like fears of receiving compassion as well as general distress disclosure tendencies. They were then enrolled in a laboratory experiment in which they recalled a personal past negative experience and were randomly assigned to write about it in a self-compassionate, self-esteem enhancing, or nondirective way. Finally, they rated how risky disclosing their experience would feel and disclosed the event in a written letter to another person. At a trait level, results indicated that the more participants feared receiving compassion, the less they tended to disclose. Moreover, selfcompassion training - but neither of the comparison conditions - significantly weakened the positive link between fear of receiving compassion and perceived risks of distress disclosure. These novel findings suggest that practicing self-compassion could help to neutralize the maladaptive relationship between fear of receiving compassion and perceived risk of disclosure.
\end{abstract} Keywords: self-compassion, self-disclosure, distress disclosure, fear of compassion 


\section{Introduction}

Self-disclosure - the process of revealing one's private thoughts, feelings, beliefs, and attitudes to others - is a common way to develop and strengthen relationships. It increases trust, intimacy, and liking between individuals when used appropriately (Collins \& Miller, 1994; Larzelere \& Huston, 1980; Laurenceau, Barrett, \& Pietromonaco, 1998). Although selfdisclosure often results in positive interpersonal outcomes, it can also have important psychological benefits, particularly when it comes to the disclosure of negative feelings. Engaging in distress disclosure - the disclosure of information regarding one's own negative experiences and emotions - to close others or to mental health professionals is related to heightened life satisfaction and subjective well-being through decreases in perceived stress and depressive symptoms (Kahn, Achter, \& Shambaugh, 2001; Saxena \& Mehrotra, 2010; Ward, Doherty, \& Moran, 2007). Distress disclosure also predicts perceived social support, which plays an important role in protecting the individual from psychosocial stress (Heinrichs, Baumgartner, Kirschbaum \& Ehlert, 2003; Hyde, Gorka, Manuck, \& Hariri, 2011; Kahn \& Hessling, 2001). Despite the apparent benefits of distress disclosure, some individuals tend to avoid disclosing their emotional distress to others, prohibiting them from seeking out social support when needed (Besser, Flett \& Davis, 2003; Richardson \& Rice, 2015). Encouraging distress disclosure, especially among those who disclose little, may be important to help them improve and/or maintain their psychological well-being.

To identify the factors that promote and deter distress disclosure, one must understand the factors that impact decisions to disclose emotional information. The perceived risk of disclosure has been proposed as a key contributor to the disclosure of intimate information (Omarzu, 2000; Vogel \& Wester, 2003). Because distress disclosure is inherently a more intimate form of 
disclosure that involves revealing one's feelings and personal insecurities to others, it is commonly perceived as a risky venture. For example, many distressing events such as personal failures or rejection experiences lead to feelings of shame which elicit fears that others will respond to the disclosure of such events with criticism and judgment (Macdonald \& Morley, 2001). Some individuals, however, may expect negative consequences from disclosure even when they expect others to be warm and compassionate rather than critical and judgmental. In particular, people who fear receiving compassion - those who feel threatened by expressions of care from others - are likely to experience anxiety or embarrassment when others show warmth or kindness (Gilbert, McEwan, Matos, \& Rivis, 2011). Gilbert et al. (2011) proposed that those who fear compassion may experience feelings of grief when others express caring towards them, as it acts as a reminder of previous instances when affection or compassion may have been withheld. Such reminders bring aversive feelings such as loneliness and yearning for close relationships to the foreground. Individuals high in fears of receiving compassion may also believe that others could use compassion as a means of manipulating them for their own personal gain (Gilbert et al., 2011). Gilbert et al. (2011) developed a measure to assess individual differences in the fear of receiving compassion and found that a greater fear of receiving compassion from others was associated with various forms of psychopathology as well as other psychosocial vulnerability factors such as self-criticism, anxious attachment, stress, and depression (Gilbert et al., 2012; Joeng \& Turner, 2015).

Thus, individuals who fear receiving compassion may perceive that sharing intimate information comes with heightened risks, potentially leading them to disclose less about their personal distress, or not at all. Although this proposed relationship between fear of receiving compassion and decreased distress disclosure has yet to be borne out empirically, avoidance of 
disclosure would be particularly concerning for those with such fears, given the heightened stress and negative affectivity to which they are vulnerable (Gilbert et al., 2011; Kelly \& Dupasquier, 2016). Thus, paradoxically, individuals high in fears of receiving compassion may be among those who would benefit most from seeking support from others through disclosure while also being among those least likely to do so.

How can those who fear receiving compassion be encouraged to trust in and accept expressions of concern and caring from others? For these individuals, practicing self-compassion may be a more palatable introduction to the experience of receiving support and kindness. Selfcompassion is a Buddhist construct defined in the psychological literature as taking a caring stance toward the self in which one aims to understand and alleviate one's own suffering without judgment (Gilbert, 2009; Neff, 2003). Neff proposed that self-compassion is comprised of three subcomponents. First, self-kindness is the most face-valid component, as it involves recognizing one's own worth and extending kindness, love, and understanding towards oneself even in the face of personal flaws. Second, mindfulness requires an acknowledgment and understanding of one's distressing emotions without becoming caught up in them to a degree that coping becomes impossible. Third, feelings of common humanity refer to the recognition that suffering is an inevitable part of being human. Whereas some individuals can feel alone or isolated in their suffering, being self-compassionate allows one to maintain feelings of connectedness to others and see one's difficulties from a broader perspective during times of distress.

Although self-compassion was originally conceptualized as being a cross-situational trait, and the majority of research to date has examined it as a stable characteristic, levels of selfcompassion can vary considerably depending on contextual factors. For example, in a daily diary study, Kelly and Stephen (2016) found that $37 \%$ of the variance in participants' levels of self- 
compassion occurred within-persons, indicating that participants' self-compassion tends to fluctuate on a day-to-day basis. Furthermore, brief experimental manipulations and interventions have been found to effectively increase state levels of self-compassion (Breines \& Chen, 2012; 2013). Thus, self-compassion demonstrates stability over time, but there is also significant variability in an individual's level of self-compassion from one period of time to the next.

Self-compassion interventions have been found to reduce distress and negative affect across individuals suffering from a variety of psychological difficulties (Barnard \& Curry, 2011). We propose that self-compassion may be particularly beneficial for individuals who fear receiving compassion both by helping them better regulate their emotions and lowering the perceived risk of disclosing their emotional experience to others (Gilbert, 2005). According to Gilbert et al. (2011), individuals who fear receiving compassion are likely to have a history of early attachment experiences during which affiliative feelings (e.g., warmth), care-seeking, and an openness to compassion were accompanied by negative outcomes such as experiences of criticism, abuse, or neglect (Gilbert et al., 2011; Miron, Seligowski, Boykin \& Orcutt, 2016). As such, these individuals learn that they are not deserving of compassion and that compassion is not to be trusted, which evidence suggests may result in a failure to develop self-compassion, leaving them less capable of regulating their own negative feelings (Kelly \& Dupasquier, 2016; Neff \& McGeeHee, 2010).

Gilbert $(2005,2014)$ proposed that self-compassion stimulates feelings of security and being cared for just as social connection and affiliation do, a theory which has received preliminary empirical support (Kelly \& Dupasquier, 2016). Individuals with heightened fears of receiving compassion from others often have similar fears of and difficulties being selfcompassionate. However, practicing self-compassion may be a less threatening starting point for 
recognizing compassion's emotional benefits, because unlike receiving compassion from others, it does not involve making oneself vulnerable to interpersonal risks like rejection. Selfcompassion can also be more easily implemented through interventions than directly receiving compassion, because the latter relies on the responses of others (see supplementary materials for further discussion). By facilitating an openness to compassion, self-compassion may reduce the impact these fears have on the perceived risk of seeking comfort from others.

The aim of the present study was two-fold. First, we sought to test the proposition that fear of receiving compassion is associated with the tendency to conceal rather than disclose distress by examining the correlation between trait measures of fear of receiving compassion and distress disclosure. Second, within the context of a laboratory-based experimental paradigm in which participants wrote about a past distressing personal experience, we investigated the impact that inducing a self-compassionate mindset would have on the relationships between fear of receiving compassion, on the one hand, and perceived risk of disclosure, negative feelings, and the length and emotional depth of the actual disclosure itself, on the other. With respect to this second aim, we tested the effects of self-compassion against two control conditions: a selfesteem enhancing condition and a free writing condition. Although self-esteem and selfcompassion are both positive self-attitudes correlated with one another, there are important distinctions between the two constructs (see Barnard \& Curry, 2011 for a review). Self-esteem is conceptualized as one's overall self-evaluation, and is characterized by self-liking and perceived competence (Rosenberg, 1965; Tafarodi \& Milne, 2006). Gilbert (2014) suggested that selfesteem and self-compassion are linked to separate affective systems, a notion supported by recent neuropsychological evidence (Simon-Thomas et al., 2012). Thus, to ensure that any observed effects were unique to self-compassion rather than a general increase in positive global 
feelings towards the self, we included a control condition aimed at enhancing self-esteem. We also included a third condition in which participants were asked to complete a non-directive "free writing" exercise to control for any beneficial effects of simply writing or thinking more about the experience (Pennebaker, 1997).

First, we hypothesized that (H1) participants who endorse greater trait-like fears of receiving compassion would report a decreased tendency to disclose distress to others. Second, practicing self-compassion should weaken the expected positive relationship between fear of receiving compassion and negative affect as well as its expected negative relationship with distress disclosure by facilitating an openness to compassion. Thus, we predicted that in the selfesteem and control conditions, fear of receiving compassion would be associated with (H2a) greater negative affect, $(\mathrm{H} 2 \mathrm{~b})$ greater perceived risk of disclosing the negative experience, and (H2c) less actual disclosure, but would be unrelated to these variables in the self-compassion condition.

\section{Method}

\section{Participants}

Participants were female undergraduate students from the psychology subject pool of a large Canadian university. They received bonus credits toward a psychology course and five dollars as remuneration. As previous research has found that both the gender of the discloser and disclosure target can have an impact on self-disclosure, only female participants were recruited (Dindia, 2002).

Out of 111 participants who signed up for the study, 90 participants completed both the online questionnaire as well as the in-lab portion of the study. Of these, five participants were excluded from analyses, four due to suspicion of deception (see procedure section below for 
explanation of funnel debriefing procedure) and one due to an inability to select a negative

experience appropriate for the study. The final sample consisted of 85 participants, ages 17-30

years old $(M=20.14, S D=2.28$; four participants did not report their age). Thirty-five $(41.2 \%)$

participants identified themselves as Caucasian, 16 (18.8\%) as East Asian, 14 (16.5\%) as South Asian, five (5.9\%) as Southeast Asian, two (2.4\%) as West Indian/Caribbean, two (2.4\%) as Middle Eastern, two (2.4\%), as Black/African, one (1.2\%) as Hispanic, and four participants (4.7\%) did not identify an ethnic background. Twenty-nine participants were in their first year of undergraduate studies (34.1\%), 13 were in their second year (15.3\%), 20 were in their third year $(23.5 \%), 15$ were in their fourth year $(17.6 \%)$, and seven were in their fifth year or above $(8.2 \%)$. One participant did not indicate their level of education. Information on participants' socioeconomic status was not collected.

\section{Procedure}

Prior to being invited into the lab for the experimental session, participants were emailed a link to complete the trait fear of receiving compassion and distress disclosure measures. The average amount of time elapsed between completion of this measure and the in-lab session was 5.09 days $(S D=3.46)$. After providing their informed consent to participate upon arrival for the experiment, participants were first asked to report their state negative affect (NA). Next, they were prompted to select a negative experience that involved feelings of failure, humiliation, or rejection but was not a trauma as defined by the Diagnostic and Statistical Manual of Mental Disorders (American Psychiatric Association, 2013; see supplementary materials for additional criteria). Participants were then asked a series of open-ended questions regarding their experience to ensure they had brought it to mind in sufficient depth (e.g., "What happened?", “When did the experience happen?", "What led up to this experience?”). Participants were then 
once again asked to report their state NA, which served as our pre-writing measure.

Subsequently, an algorithm in Qualtrics ${ }^{\mathrm{TM}}$ randomly assigned participants to complete one of three writing exercises that served as the experimental manipulation: (a) a selfcompassion exercise, (b) a self-esteem enhancing exercise, or (c) a free writing exercise, each modeled after the writing exercises developed by Leary, Tate, Allen, Adams, and Hancock (2007, study 5) and used in previous studies manipulating self-compassion (Breines \& Chen, 2012; Johnson \& O’Brien, 2013; Odou \& Brinker, 2015). In each condition, participants were asked to respond to three writing prompts. They could write for as long as they chose, but were told the exercise should take approximately 10 minutes. All participants were informed that the aim of the writing exercise was to alleviate negative feelings regarding their negative experience.

Experimental manipulations. In the self-compassion condition, the prompts were designed to target feelings related to the three components of self-compassion as defined by Neff (2003): (a) self-kindness (i.e., “...write a paragraph expressing kindness, understanding, and concern toward yourself”), (b) mindfulness (i.e., “...write about the event in a detached, objective fashion”), and (c) common humanity (i.e., “...write down ways in which other people also experience events that are similar to the one you described"). In the self-esteem condition, the prompts were designed to either boost or preserve the participant's self-esteem by: (a) focusing on personal strengths (b) making defensive attributions (Maltese, Alesi, \& Alù, 2012) and (c) remembering past successes. The free writing condition prompts were designed to account for the impact that simply writing and thinking about the experience could have on participants' feelings (Pennebaker, 1997). In this condition, participants were instructed to “...really let go" and explore their deepest (a) thoughts, (b) feelings, and (c) beliefs about the experience. See supplementary materials for complete writing prompts. After completing the 
writing exercise, participants were once again asked to complete the NA items, which served as their post-writing exercise score.

Deception. The researcher then informed the participants that, as another means of improving their feelings regarding their negative experience, they would have the chance to engage in a supportive discussion with another female participant. Participants were told that each of them would be asked to write a letter to one another describing their negative experiences, exchange their letters, and afterwards they would come together to discuss. They were told this procedure was necessary to ensure that there was no mutual influence on their level of disclosure. Researchers emphasized that participants could share as much or as little as they wished, or nothing at all if desired. They were also informed that prior to exchanging letters, the researcher would verify that the other female participant was unknown to them, and they would have a chance to withdraw their letter if they knew their conversation partner and were uncomfortable proceeding.

Prior to writing their letter, participants were asked to complete a measure of the perceived risk of disclosing to the other participant. Then, participants completed their letter to the supposed other participant, after which the study was terminated. None of the letters were actually read by other participants, and no supportive conversation took place. Researchers conducted a funnel debriefing procedure to probe for suspicion regarding deception and the purpose of the study. Participants who fully doubted the deception were excluded from analyses (see participant section above). Finally, participants were debriefed and given the opportunity to raise any questions or concerns. At this stage, we obtained informed consent to use participants' data with their complete awareness of the study's true purposes.

\section{Measures}


All questionnaires were administered via Qualtrics ${ }^{\mathrm{TM}}$, a US-based online survey tool.

Previous disclosure regarding the negative experience. To examine whether participants were selecting negative experiences that they had not previously disclosed in depth, they were asked to respond to a single item, "How much have you shared about your thoughts and feelings regarding this negative experience with others?" on a Likert-type scale from 1 (“I have very slightly shared my thoughts and feelings about this experience, or have not shared them at all") to 5 ("I have shared my thoughts and feelings about this experience in full and complete detail").

Perceived severity of the negative experience. To verify that participants across conditions were selecting negative experiences of similar subjective severity, they were asked to respond to the question "Right at this moment, how badly does this experience make you feel about yourself?" on a visual analogue scale ranging from 0 ("Not at all") to 100 ("Very badly").

Effort. To ensure that participants were sufficiently engaged in the experimental manipulation, participants were asked to respond to a single item, "How much effort did you honestly apply to the written exercise?" on a 5-point Likert-type scale from 1 ("No effort/did not do it") to 5 ("All of the effort that I was able to").

Fear of receiving compassion. The 13-item section from the Fears of Compassion Scales developed by Gilbert et al. (2011) was used to assess fears of compassion from others (e.g., "I try to keep my distance from others even if I know they are kind", "Feelings of kindness from others are somehow frightening"). Using a five-point Likert scale ranging from 0 ("strongly disagree") to 4 ("strongly agree"), participants are asked to indicate the extent to which they agree with a set of compassion-related statements. Participants' totals have a minimum possible score of 0 and a maximum possible score of 52. Cronbach's alpha was .90 in the current study, 
indicating excellent internal consistency.

Distress disclosure. The Distress Disclosure Index (DDI) was developed by Kahn and Hessling (2001) to measure the tendency to conceal versus disclose psychological distress. On a scale from 1 ("strongly disagree") to 5 ("strongly agree"), participants rated their agreement with 12 items regarding their typical level of disclosure to close others (e.g., "When I feel upset, I usually confide in my friends", "I usually seek out someone to talk to when I am in a bad mood"). Total scores are calculated by taking the average of the 12 item scores, where greater scores indicate a greater tendency to dislose distress to others. A review of previous research demonstrated the DDI to be a highly reliable instrument with alpha coefficients ranging from .89 to .95 (Kahn, Hucke, Bradley, Glinski, \& Malak, 2012). The measure's internal consistency was equally excellent in the present study (Cronbach's alpha $=.96)$.

Negative affect. State negative affect (NA) was measured using two visual analogue scale items: (a) "upset" and (b) "distressed". On a scale represented by a slider ranging from 0 ("Very slightly or not at all") to 100 ("Extremely"), participants were asked to indicate the degree to which they felt each emotion at the present moment. Responses to both items were averaged to create a composite measure of NA. Affect was measured at three time points: (T1) prior to the negative experience recall, (T2) after recalling their negative experience but before completing the writing exercise, and (T3) post-writing exercise. Spearman-Brown coefficients for the NA items in the present study were .76 pre-writing and .85 post-writing exercise.

Perceived risk. To measure the perceived risk of making a disclosure to the other participant in the study, the risk-related items from Vogel and Wester's Disclosure Expectations Scale (2003) were administered. Participants were asked to respond to the four Likert-style items (e.g., "How risky does it feel to disclose your negative experience to the other participant?", 
"How difficult will it be for you to disclose personal information to the other participant?") on a scale from 1 ("Not at all") to 7 ("Very"). The average of these four items was used as a composite measure of perceived risk of disclosure. Cronbach's alpha for the four items was .82 in the present study, indicating good internal consistency.

Letter content (LIWC2015). As an objective measure of disclosure length and emotional depth, the text analysis software program Linguistic Inquiry and Word Count: 2015 (LIWC2015) was used to analyze the content of the letters participants wrote to the alleged other participant. LIWC2015 counts word appearances and can classify them into a range of different categories using dictionaries of approximately 6,400 words and word stems (Pennebaker, Boyd, Jordan \& Blackburn, 2015). In addition to total word count, the linguistic category of negative emotion was used to count emotion-related word appearances, calculated as a proportion of the total word count. Proportion of negative words has been used as a measure of disclosure depth in previous research (Callaghan, Graff, \& Davies, 2013; Houghton \& Joinson, 2012).

\section{Data Analyses}

Analyses were conducted in IBM SPSS Statistics 20 (2011). Hierarchical multiple regression was used to investigate whether experimental condition moderated the associations between fear of receiving compassion and our outcome variables of interest. At the first step, fear of receiving compassion was entered (grand mean centered), followed by two dummy-coded variables at the second step representing the main effect of condition. Finally, two interaction terms were entered at the third step to represent the condition by fear of receiving compassion interaction. The main dependent variables were post-writing (T3) NA, perceived risk of disclosure, and actual disclosure as measured by letter word count and the proportion of negative emotion words used in participants' letters (as measured by LIWC2015). Significant interactions 
were interpreted by examining simple slopes (the conditional effect of fear of receiving compassion in step 3 for the condition represented with a zero in each of the dummy-coded contrasts in the model).

\section{Results}

\section{Relationship Between Fear of Receiving Compassion and Distress Disclosure}

The zero-order correlation between fear of receiving compassion and the Distress Disclosure Index indicated the presence of a significant negative relationship $(r(83)=-.49, p<$ .001). This provided support for our hypothesis that fear of receiving compassion would be associated with decreased disclosure of negative feelings and distressing experiences to others.

\section{Equivalence of Groups}

Of the 85 participants included in the analyses, 29 were randomly assigned to the selfcompassion condition, 30 were assigned to the self-esteem condition, and 26 were assigned to the free writing condition. There were no significant differences between conditions on any demographic variables or baseline measures (see Table 1 for means and standard deviations, see supplementary materials for analyses).

\section{Zero-Order Correlations}

Zero-order correlations were calculated to examine relationships between proposed predictor and outcome variables. As shown in Table 2, results demonstrated that individuals with heightened fears of receiving compassion felt worse about themselves due to their past negative experience and had higher pre-writing exercise (T2) NA. Perceived risk of disclosure was positively related to how badly participants felt about themselves regarding the event and to their NA. Letter word count was negatively related to perceived risk of disclosure, suggesting that those who felt that disclosure would be more risky disclosed less in their letter to the other 
participant. Participants whose letters contained a higher proportion of NA words composed shorter letters overall and perceived the disclosure of their negative experience to be riskier. Finally, participants' general distress disclosure tendencies did not predict NA, perceived risk of disclosure, or actual disclosure.

\section{Manipulation Checks of Engagement and Effort}

To examine whether participants were emotionally engaged during the recall of their distressing experience, a repeated measures ANOVA was conducted to examine changes in NA from before (T1) to after (T2) bringing their negative experience to mind. Analyses revealed a main effect of time, which indicated that NA increased from pre- to post-recall $(F(1,82)=$ $\left.103.82, p<.001, \eta_{p}{ }^{2}=.56\right)$. No significant time by condition interaction was found $(F(2,82)=$ $\left..48, p=.62, \eta_{p}{ }^{2}=.01\right)$. These results suggest that participants were emotionally engaged in the recall activity across conditions.

A one-way ANOVA indicated there was no difference in self-reported effort applied across conditions $\left(F(1,82)=.82, p=.44 \eta_{p}^{2}=.02\right)$. The overall mean rating of effort applied during the writing exercise was 3.65 out of $5(S D=.84)$, suggesting that participants applied themselves reasonably well to their assigned writing exercise.

\section{Condition as a Moderator of Associations Between Fear of Receiving Compassion and}

\section{Outcomes}

In the analysis of negative affect, $\mathrm{T} 2$ scores were entered as a covariate in the hierarchical linear regression to control for pre-writing levels of NA. No significant main effect of condition was present (see Table $3 \Delta R^{2}$ for step 2). Consistent with our hypotheses, there was a significant interaction between condition and fear of receiving compassion. This interaction term accounted for $6.4 \%$ of the variance in post-writing (T3) NA (see $\Delta R^{2}$ for step 3). The slope for fear of 
receiving compassion predicting NA in the self-compassion condition was significantly different from the slopes for participants in the self-esteem and free writing conditions (as indicated by the tests of the two interaction terms entered in step 3). An identical analysis using a third dummy code to replace D1 (D3: self-compassion $=1$, self-esteem $=0$, free writing $=0$ ) revealed that the slopes of the self-esteem and free writing conditions did not differ significantly from one another $(B=.23, S E=.53, \beta=.05, p=.66)$. An examination of the simple slopes using pre-writing (T2) NA scores as a covariate revealed that there was a significant positive relationship between NA post-writing exercise (T3) and fears of receiving compassion in the free writing condition $(B=$ $.84, S E=.37, p=.03,95 \%$ CI $\left.[.10,1.58], s r^{2}=.034\right)$, a non-significant positive relationship in the self-esteem condition $\left(B=.61, S E=.39, p=.12,95 \% \mathrm{CI}[-.16,1.38], s r^{2}=.016\right)$, and a non-significant negative relationship in the self-compassion condition $(B=-.40, S E=.26, p=$ $.13,95 \%$ CI [-.92. .12], $s r^{2}=.016$; see Figure 1).

Results from the analysis of perceived risk ratings indicated that although there was no significant main effect of condition (see Table $3, \Delta R^{2}$ for step 2), there was a significant interaction between condition and fear of receiving compassion, consistent with our hypotheses. This interaction accounted for $7.4 \%$ of the variance in risk of disclosure ( $\Delta R^{2}$ for step 3$)$. The slope for fear of receiving compassion predicting perceived risk in the self-compassion condition was significantly different from the slopes for participants in the self-esteem and free writing conditions (as indicated by the tests of both interaction terms entered in step 3). An identical analysis using a third dummy code to replace D1 (D3: self-compassion $=1$, self-esteem $=0$, free writing $=0$ ) verified that the slopes of the self-esteem and free writing conditions did not differ significantly from one another $(B=-.002, S E=.03, \beta=-.01, p=.94)$. An examination of the simple slopes supported our hypotheses and revealed a non-significant positive relationship 
between fears of receiving compassion and perceived risk of disclosure in the self-esteem $(B=$ $\left..04, S E=.03, p=.08,95 \% \mathrm{CI}[-.01, .09], s r^{2}=.036\right)$ and free writing $(B=.04, S E=.02, p=$ $.09,95 \%$ CI $\left.[-.01, .09], s r^{2}=.034\right)$ conditions, and a weaker, non-significant negative relationship in the self-compassion condition $\left(B=-.02, S E=.02, p=.27,95 \%\right.$ CI [-.05, .02], $s r^{2}$ $=.014$; see Figure 2).

Separate hierarchical linear regressions were conducted on each of the LIWC2015 disclosure outcome variables. No significant main effect of condition or condition by fear of compassion interaction was found for letter word count $\left(\right.$ step $2 \Delta R^{2}=.01, \Delta F(2,81)=.40, p=$ .67 ; step $\left.3 \Delta R^{2}=.01, \Delta F(2,79)=.39, p=.68\right)$. However, there was a marginally significant main effect of condition on proportion of negative affect words ( $\operatorname{step} 2 \Delta R^{2}=.07, \Delta F(2,80)=$ $3.15, p=.05)$. Effects of the dummy coded contrasts revealed that letters of participants in the free writing condition contained a greater proportion of NA words than those of participants in the self-compassion condition $(3.6 \%$ versus $2.7 \%$, respectively; $B=.36, S E=.15, p=.02,95 \%$ CI $\left.[.06, .65], s r^{2}=.068\right)$. Letters of participants in the self-esteem condition did not differ significantly in their proportion of negative words from those in the self-compassion or free writing conditions $\left(B=.23, S E=.14, p=.11,95 \%\right.$ CI $[-.05, .52], s r^{2}=.032 ; B=.12, S E=.14$, $p=.40,95 \%$ CI $[-.16, .41], s r^{2}=.009$, respectively). Contrary to hypotheses, this effect was not qualified by a fear of compassion by condition interaction (step $3 \Delta R^{2}=.001, \Delta F(2,78)=.03, p$ $=.97)$.

The analyses reported above were also conducted with perceived severity of the selected event as a control variable/covariate. Perceived severity was only found to be a significant predictor for perceived risk of disclosure, although the pattern and interpretation of the results were unaffected (see Supplementary Table 1). 


\section{Discussion}

The current study had two overarching objectives. Our first aim was to test the hypothesis that individuals with heightened fears of receiving compassion would report a greater overall reluctance to disclose distressing experiences to others. Our second aim was to determine whether practicing self-compassion would attenuate the relationship between fear of receiving compassion and the perceived risk of - and tendency toward - disclosing that experience to someone else.

Results indicated there was a moderate to large negative correlation between fear of receiving compassion and the tendency to disclose experiences of distress to others at a trait level, supporting hypothesis 1 . Individuals who were more concerned about or untrusting of receiving compassion from others were also less likely to report seeking out others with whom they can share their feelings when they are suffering or upset.

Consistent with hypotheses $2 \mathrm{a}$ and $2 \mathrm{~b}$, fear of receiving compassion interacted with condition to predict both NA and perceived risk of disclosure. Despite this significant interaction, the conditional slopes for the most part did not reach statistical significance, which may indicate that although the effects of fear of receiving compassion differed between our control conditions and the self-compassion condition, our study was underpowered to detect the small effect sizes found here. For example, the squared semi-partial correlations for the simple slopes indicated that fears of receiving compassion accounted for approximately 3.4 to $3.6 \%$ of the variance in perceived risk of disclosure in the self-esteem and free writing conditions, respectively. This translates to a small Cohen's $f^{2}$ of approximately .038. Future attempts to replicate the current findings should recruit larger sample sizes to ensure adequate power (e.g., $N$ $=165$ to achieve a power of .80 for a Cohen's $f^{2}$ of .038). Given these issues, we proceed by 
cautiously interpreting the direction of the conditional slopes.

Whereas fear of receiving compassion was positively related to post-writing (T3) NA and perceived risk for participants in the self-esteem and free writing conditions, fear of receiving compassion was negatively or unrelated to post-writing NA and perceived risk for participants in the self-compassion condition. Together, these findings suggest that by writing about their past negative experiences self-compassionately, the strength of the relations between participants' fears of receiving compassion and their levels of NA and perceived risk of disclosure were weakened considerably or even reversed. Although we did not assess for possible mechanisms underlying this effect, a number of plausible explanations exist. In our sample, fear of receiving compassion was positively correlated with pre-writing (T2) NA, which is consistent with previous findings that those who fear compassion tend to experience more distress than others (Cunha, Pereira, Galhardo, Couto, \& Massano-Cardoso, 2015; Gilbert et al., 2012). Thus, these individuals would be expected to benefit most from interventions aimed at regulating negative affect by nurturing feelings of caring and warmth that they have previously avoided. Moreover, as self-compassion has been linked to feeling more secure and connected to others within one's social world, practicing self-compassion might have led these individuals to feel safer, less threatened, and thereby more trusting of others, loosening the connection between their fears and the perceived risks associated with self-disclosure (Kelly \& Dupasquier, 2016).

Finally, although participants in the self-compassion condition used a smaller proportion of NA-laden words in their disclosure letters than those in the free writing condition, our results did not support that condition would moderate the relationship between fear of receiving compassion and the characteristics of participants' actual disclosures (hypothesis 2c). One possible explanation for this finding is that priming self-compassion truly does not facilitate 
distress disclosure regardless of one's level of discomfort with receiving care from others. It is also possible that other factors obscured the real impact of cultivating self-compassion on actual disclosure. For example, as participants in the self-compassion condition experienced the least NA after their assigned writing exercise, they might have felt less need to disclose regarding negative emotions. Previous research has demonstrated that willingness to disclose and the quality of disclosures depend both on the perceived risk of disclosure and utility of making a disclosure (Omarzu, 2000; Vogel \& Wester, 2003). It is possible that, by engaging in the writing intervention after recalling their negative experience, the reduction in distress that participants experienced also led to a relative reduction in the perceived utility of making a disclosure (i.e., if one is less distressed, further reduction of distress through disclosure may be unnecessary). This may also explain why, contrary to what theory would predict, fear of receiving compassion was uncorrelated with actual disclosure at a zero-order level. In the context of the study where participants were explicitly told that disclosure could be useful for them, those who fear receiving compassion could have simultaneously perceived that the disclosure would be risky and helpful, resulting in no observed zero-order relationship between fear of receiving compassion and actual disclosure. Furthermore, the relatively contrived environment offered by writing a letter rather than speaking directly to another participant might have dampened the relationship between perceived risk and disclosure. Disclosure via letter writing is inherently characterized by a lack of genuine reciprocity, which may be an important part of the trustbuilding process that facilitates longer and deeper self-disclosures - a process that may be most reliably measured in naturalistic rather than experimental contexts.

Nonetheless, fear of receiving compassion positively predicted NA as well as risk of disclosure within the self-esteem and free writing conditions, suggesting that the more afraid 
individuals are of receiving compassion, the less responsive they may be to psychological interventions designed to reframe distressing personal experiences. However, interventions that focus on enhancing self-compassion may be an exception. Teaching such individuals to become more self-compassionate could uncouple their fears from the risks associated with opening up to others and allow additional factors to be taken into consideration when making a disclosure, such as its potential benefits. On a conscious level, practicing self-compassion might trigger the realization that compassion - be it from themselves or from others - may actually be helpful, which may encourage disclosure to others. Further research is needed to determine whether this approach might encourage more active help-seeking behavior, such as calling a friend for support or making an appointment to see a therapist. If future research demonstrates that selfcompassion interventions can in fact increase disclosure of distressing events, there could be valuable clinical applications. As in-session disclosure has been found to be a predictor of therapeutic success, encouraging self-concealing clients to share their feelings and experiences more openly through self-compassion-based interventions may facilitate treatment response (Sloan \& Kahn, 2005).

\section{Limitations and Future Directions}

The present study has a number of limitations. First, our sample was restricted to female undergraduate participants only. Thus, we have little information on the generalizability of the current findings to other populations. Future studies should seek to replicate findings with community and male participants as well as mixed-gender dyads. It would also be important to examine whether the current results would generalize to a clinical sample, where the fear of receiving compassion from others is likely to be especially high. It may be that recalling negative experiences would have a far more toxic effect in such a population and that a longer, therapist- 
led intervention would be required to achieve the same effects observed in the present study.

Second, the current study's design placed fairly strict constraints on the types of events participants could select both in order to ensure they were choosing comparable experiences and to minimize possible harm caused to them by recalling more traumatic experiences. Although the instructions encouraged participants to select a negative experience that was personally meaningful to them, it is possible that they prevented participants from choosing experiences they desired to conceal most from others or that involved more intense distress. In fact, trauma is the type of experience that individuals might benefit most from disclosing to loved ones, clinicians, or other professionals. Thus, future research should aim to investigate whether the present results generalize to more intense or threatening experiences such as trauma or abuse.

Third, the design of the current study placed participants in a rather contrived disclosure situation, which could have lowered ecological validity. The processes at play in this experimental context may not be generalizable to more naturalistic settings. To build on our research, future studies might look at whether being more self-compassionate on a given day is associated with greater daily disclosure of distressing events in the context of participants' actual lives, especially among those who strongly fear receiving compassion.

Furthermore, as discussed above, it is possible that the writing intervention might have reduced some participants' distress to a degree that disclosure may no longer have been viewed as helpful or useful, thus decreasing the motivation to disclose. To circumvent this issue, future studies would benefit from a careful consideration of the timing of self-compassion interventions. For example, implementing an intervention designed to increase general selfcompassion and recording disclosure of subsequent negative experiences may be a methodologically sound alternative to targeting the negative experience using self-compassion 
after the fact. The present study also entailed very brief interventions with no follow-up assessment of their long-term impact. Thus, future intervention studies should aim to implement a longer-term self-compassion intervention such as compassionate imagery training (Gilbert \& Irons, 2004), and examine its impact over a subsequent number of weeks.

Finally, fear of compassion from others is strongly related to anxious attachment $(r=.74$; Gilbert et al., 2011). In fact, fears of compassion are thought to develop in part from insecure attachments to early caregivers, where support would have been withheld or provided inconsistently when needed (Joeng et al., 2017; Kelly \& Dupasquier, 2016). However, whereas fear of receiving compassion encompasses emotional reactions to expressions of kindness in particular, insecure attachment is a much broader construct. Nonetheless, future research should incorporate measures of both constructs in order to examine which is more strongly related to the perceived risks of distress disclosure and whether self-compassion training might be beneficial not only for those with fears of receiving compassion but also for those with deeper relational insecurities.

\section{Ethical Approval}

All procedures performed in studies involving human participants were in accordance with the ethical standards of the institutional and/or national research committee and with the 1964 Helsinki declaration and its later amendments or comparable ethical standards. 


\section{References}

American Psychiatric Association. (2013). Diagnostic and statistical manual of mental disorders (5th ed.). Washington, DC: Author.

Barnard, L.K., \& Curry, J.F. (2011). Self-compassion: Conceptualizations, correlates, \& interventions. Review of General Psychology, 15, 289-303.

Besser, A., Flett, G.L., \& Davis, R.A. (2003). Self-criticism, dependency, silencing the self, and loneliness: A test of a mediational model. Personality and Individual Differences, 35, 1735-1752.

Breines, J.G., \& Chen, S. (2012). Self-compassion increases self-improvement motivation. Personality and Social Psychology Bulletin, 38, 1133-1143.

Breines, J., \& Chen, S. (2013). Activating the inner caregiver: The role of support-giving schemas in increasing state self-compassion. Journal of Experimental Social Psychology, 49, 58-64.

Callaghan, D.E., Graff, M.F., \& Davies, J. (2013). Revealing all: Misleading self-disclosure rates in laboratory-based online research. Cyberpsychology, Behaviour, and Social Networking, 16, 690-694.

Collins, N.L., \& Miller, L.C. (1994). Self-disclosure and liking: A meta-analytic review. Psychological Bulletin, 116, 457-475.

Cunha, M., Pereira, C., Galhardo, A., Couto, M., \& Massano-Cardoso, I. (2015). Social anxiety in adolescents: The role of early negative memories and fear of compassion. European Psychiatry, 30, 28-31.

Dindia, K. (2002). Self-disclosure research: Knowledge through meta-analysis. In M. Allen, R.W. Preiss, B.M. Gayle \& N.A. Burrell (Eds.), Interpersonal communication research: 
Advances through meta-analysis (pp. 169-185). Mahwah, N.J.: Lawrence Erlbaum Associates.

Gilbert, P. (Ed.). (2005). Compassion: Conceptualization, research, and use in psychotherapy. London: Routledge.

Gilbert, P. (2009). Introducing compassion-focused therapy. Advances in Psychiatric Treatment, $15,199-208$.

Gilbert, P. (2014). The origins and nature of compassion focused therapy. The British Journal of Clinical Psychology, 53, 6-41.

Gilbert, P. \& Irons, C. (2004). A pilot exploration of the use of compassionate images in a group of self-critical people. Memory, 12, 507-516.

Gilbert, P., McEwan, K., Gibbons, L., Chotai, S., Duarte, J., \& Matos, M. (2012). Fears of compassion and happiness in relation to alexithymia, mindfulness, and self-criticism. Psychology and Psychotherapy: Theory, Research and Practice, 85, 374-390.

Gilbert, P., McEwan, K., Matos, M., \& Rivis, A. (2011). Fears of compassion: Development of three self-report measures. Psychology and Psychotherapy: Theory, Research, and Practice. 84, 239-255.

Heinrichs, M., Baumgartner, T., Kirschbaum, C., \& Ehlert, U. (2003). Social support and oxytocin interact to suppress cortisol and subjective responses to psychosocial stress. Biological Psychiatry, 54, 1389-1398.

Houghton, D.J., \& Joinson, A.N. (2012, January). Linguistic markers of secrets and sensitive self-disclosure in Twitter. Paper presented at the 45th Hawaii International Conference on System Sciences, Maui, HI.

Hyde, L.W., Gorka, A., Manuck, S.B., \& Hariri, A.R. (2011). Perceived social support 
moderates the link between threat-related amygdala reactivity and trait anxiety. Neuropsychologia, 49, 651-656.

IBM Corporation. (2011). IBM SPSS Statistics for Windows, Version 20.0. Armonk, NY: IBM Corporation.

Joeng, J.R. \& Turner, S.L. (2015). Mediators between self-criticism and depression: Fear of compassion, self-compassion, and importance to others. Journal of Counselling Psychology, 62, 453-463.

Joeng, J.R., Turner, S.L., Kim, E.Y., Choi, S.A., Lee, Y.J., Kim, J.K. (2017). Insecure attachment and emotional distress: Fear of self-compassion and self-compassion as mediators. Personality and Individual Differences, 112, 6-11.

Johnson, E.A., \& O’Brien, K.A. (2013). Self-compassion soothes the savage EGO-threat system: Effects on negative affect, shame, rumination, and depressive symptoms. Journal of Social and Clinical Psychology, 32, 939-963.

Kahn, J.H., Achter, J.A., \& Shambaugh, E.J. (2001). Client distress disclosure, characteristics at intake, and outcome in brief counseling. Journal of Counseling Psychology, 48, 203-211.

Kahn, J.H., \& Hessling, R.M. (2001). Measuring the tendency to conceal versus disclose psychological distress. Journal of Social and Clinical Psychology, 20, 41-65.

Kahn, J.H., Hucke, B.E., Bradley, A.M., Glinski, A.J., \& Malak, B.L. (2012). The Distress Disclosure Index: A research review and multi-trait-multimethod examination. Journal of Counseling Psychology, 59, 134-149.

Kelly, A.C. \& Dupasquier, J. (2016). Social safeness mediates the relationship between recalled parental warmth and the capacity for self-compassion and receiving compassion. Personality and Individual Differences, 89, 157-161. 
Kelly, A.C. \& Stephen, E. (2016). A daily diary study of self-compassion, body image, and eating behavior in female college students. Body Image, 17, 152-160.

Larzelere, R.E., \& Huston, R.L. (1980). The Dyadic Trust Scale: Toward understanding interpersonal trust in close relationships. Journal of Marriage and Family, 42, 595-604.

Laurenceau, J.P., Barrett, L.F., \& Pietromonaco, P.R. (1998). Intimacy as an interpersonal process: The importance of self-disclosure, partner disclosure, and perceived partner responsiveness in interpersonal exchanges. Journal of Personality and Social Psychology, 74, 1238-1251.

Leary, M.R., Tate, E.B., Allen, A.B., Adams, C.E., \& Hancock, J. (2007). Self-compassion and reactions to unpleasant self-relevant events: The implications of treating oneself kindly. Journal of Personality and Social Psychology, 5, 887-904.

Macdonald, J., \& Morley, I. (2001). Shame and non-disclosure: A study of the emotional isolation of people referred for psychotherapy. British Journal of Medical Psychology, 74(1), 1-21.

Maltese, A., Alesi, M, \& Alù, A.G. (2012). Self-esteem, defensive strategies and social intelligence in adolescence, Procedia - Social and Behavioral Sciences, 69, 2054-2060.

Miron, L.R., Seligowski, A.V., Boykin, D.M., \& Orcutt, H.K. (2016). The potential indirect effect of childhood abuse on posttrauma pathology through self-compassion and fear of self-compassion. Mindfulness, 7, 596-605.

Neff, K.D. (2003). The development and validation of a scale to measure self-compassion. Self and Identity, 2, 223-250.

Neff, K.D. \& McGeehee, P. (2010). Self-compassion and psychological resilience among adolescents and young adults. Self and Identity, 9, 225-240. 
Odou, N. \& Brinker, J. (2015). Self-compassion, a better alternative to rumination than distraction as a response to negative mood. The Journal of Positive Psychology, 10, 447457.

Omarzu, J. (2000). A disclosure decision model: Determining how and when individuals will self-disclose. Personality and Social Psychology Review, 2, 174-185.

Pennebaker, J.W. (1997). Writing about emotional experiences as a therapeutic process. Psychological Science, 8, 162-166.

Pennebaker, J.W., Boyd, R.L., Jordan, K., \& Blackburn, K. (2015). The development and psychometric properties of LIWC2015. Austin, TX: University of Texas at Austin.

Richardson, C.M. \& Rice, K.G. (2015). Self-critical perfectionism, daily stress, and disclosure of emotional events. Journal of Counseling Psychology, 62, 694-702.

Rosenberg, M. (1965). Society and the adolescent self-image. Princeton, NJ: Princeton University Press.

Saxena, P. \& Mehrotra, S. (2010). Emotional disclosure in day-to-day living and subjective wellbeing. Psychological Studies, 55, 208-218.

Simon-Thomas, E.R., Godzik, J., Castle, E., Antonenko, O., Ponz, A., Kogan, A., \& Keltner, D.J. (2012). An fMRI study of caring vs self-focus during induced compassion and pride. Social Cognitive and Affective Neuroscience, 7, 635-648.

Sloan, A.E., \& Kahn, J.H. (2005). Client self-disclosure as a predictor of short-term outcome in brief psychotherapy. Journal of College Student Psychotherapy, 19(3), 25-39.

Tafarodi, R.W., \& Milne, A.B. (2006). Decomposing global self-esteem. Journal of Personality, $70,443-483$.

Vogel, D.L., \& Wester, S.R. (2003). To seek help or not to seek help: The risks of self- 
disclosure. Journal of Counseling Psychology, 3, 351-361.

Ward, M., Doherty, D.T., \& Moran, R. (2007). It's good to talk: Distress disclosure and psychological wellbeing. Dublin, Ireland: Health Research Board. 


\section{Tables}

Table 1

Means and standard deviations of all variables

\begin{tabular}{|c|c|c|c|c|c|c|c|c|c|c|c|c|}
\hline & \multicolumn{6}{|c|}{ Pre-writing exercise (T2) } & \multicolumn{6}{|c|}{ Post-writing exercise (T3) } \\
\hline & \multicolumn{2}{|c|}{$\begin{array}{l}\text { Self-compassion } \\
\text { condition }\end{array}$} & \multicolumn{2}{|c|}{$\begin{array}{l}\text { Self-esteem } \\
\text { condition }\end{array}$} & \multicolumn{2}{|c|}{$\begin{array}{l}\text { Free writing } \\
\text { condition }\end{array}$} & \multicolumn{2}{|c|}{$\begin{array}{l}\text { Self-compassion } \\
\text { condition }\end{array}$} & \multicolumn{2}{|c|}{$\begin{array}{l}\text { Self-esteem } \\
\text { condition }\end{array}$} & \multicolumn{2}{|c|}{$\begin{array}{l}\text { Free writing } \\
\text { condition }\end{array}$} \\
\hline & Mean & $S D$ & Mean & $S D$ & Mean & $S D$ & Mean & $S D$ & Mean & $S D$ & Mean & $S D$ \\
\hline $\begin{array}{l}\text { Fear of receiving } \\
\text { compassion }\end{array}$ & 17.00 & 11.97 & 14.83 & 7.96 & 13.66 & 8.88 & - & - & - & - & - & - \\
\hline Trait distress disclosure & 2.92 & 1.17 & 3.12 & .91 & 3.50 & .97 & - & - & - & - & - & - \\
\hline Previous disclosure & 2.38 & 1.12 & 2.13 & 1.14 & 2.08 & .98 & - & - & - & - & - & - \\
\hline $\begin{array}{l}\text { Event related negative self- } \\
\text { feelings }\end{array}$ & 61.52 & 24.01 & 61.50 & 17.87 & 55.92 & 22.04 & - & - & - & - & - & - \\
\hline Negative affect & 45.79 & 25.89 & 38.58 & 21.01 & 37.65 & 19.20 & 24.83 & 23.73 & 24.85 & 19.25 & 30.19 & 22.94 \\
\hline Perceived risk of disclosure & - & - & - & - & - & - & 4.01 & 1.16 & 3.87 & 1.02 & 3.73 & 1.13 \\
\hline Letter word count & - & - & - & - & - & - & 163.52 & 84.65 & 167.17 & 72.70 & 176.88 & 86.22 \\
\hline $\begin{array}{l}\text { Proportion of negative } \\
\text { affect words in letter }\end{array}$ & - & - & - & - & - & - & .027 & .019 & .032 & .019 & .036 & .016 \\
\hline
\end{tabular}

Note: Only NA was assessed both pre- (T2) and post-writing exercise (T3) 
Table 2

Zero-order correlations between predictor and outcome variables

$\begin{array}{llllll}1 & 2 & 3 & 4 & 5 & 6\end{array}$

1. Fear of receiving compassion

2. Trait distress disclosure

$-.49 * * \quad-$

3. Event-related negative self-feelings

$.22 * \quad-.02$

4. Negative affect (T2)

$.29 * * \quad .05 \quad .55 * *$

5. Perceived risk of disclosure

$.11 \quad .05 \quad .22 * \quad .23 *$

6. Letter word count

$\begin{array}{lllll}.20 \dagger & -.05 & .09 & .02 & -.25^{*}\end{array}$

7. Proportion of negative affect words in letter $\begin{array}{lllllll}.03 & & .08 & .15 & .05 & .23 * & -.24 *\end{array}$ $\dagger p<.10, * p<.05, * * p<.01$ 
Table 3

Hierarchical linear regressions in which fear of receiving compassion interacts with condition to predict post-writing (T3) negative affect and perceived risk of disclosure

\begin{tabular}{|c|c|c|c|c|c|c|c|c|c|c|}
\hline & \multicolumn{5}{|c|}{ Negative affect } & \multicolumn{5}{|c|}{ Perceived risk of disclosure } \\
\hline & $B$ & $S E$ & $\beta$ & $\Delta R^{2}$ & $\Delta F$ & $B$ & $S E$ & $\beta$ & $\Delta R^{2}$ & $\Delta F$ \\
\hline Step 1 & & & & .002 & .22 & & & & .013 & 1.06 \\
\hline Pre-writing (T2) negative affect & .59 & .09 & $.60 * *$ & & & - & - & - & - & \\
\hline Fear of receiving compassion & .10 & .20 & .04 & & & .01 & .01 & .11 & & \\
\hline Step 2 & & & & .039 & $2.69 \dagger$ & & & & .008 & .33 \\
\hline Pre-writing (T2) negative affect & .61 & .09 & $.63 * *$ & & & - & 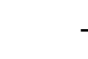 & - & - & - \\
\hline Fear of receiving compassion & .14 & .20 & .06 & & & .01 & .01 & .10 & & \\
\hline D1 & 4.76 & 4.49 & .10 & & & -.12 & .29 & -.05 & & \\
\hline D2 & 10.84 & 4.68 & $.23 *$ & & & -.25 & .30 & -.10 & & \\
\hline Step 3 & & & & .064 & $4.81 *$ & & & & .074 & $3.24 *$ \\
\hline Pre-writing (T2) negative affect & .60 & .08 & $.62 * *$ & & & - & - & - & - & \\
\hline Fear of receiving compassion & -.40 & .26 & -.18 & & & -.02 & .02 & -.17 & & \\
\hline D1 & 3.90 & 4.30 & .09 & & & -.16 & .28 & -.07 & & \\
\hline D2 & 10.88 & 4.49 & $.23^{*}$ & & & -.25 & .29 & -.11 & & \\
\hline D1 x Fear of receiving compassion & 1.01 & .46 & $.22 *$ & & & .06 & .03 & $.27 *$ & & \\
\hline D2 $x$ Fear of receiving compassion & 1.24 & .45 & $.28 * *$ & & & .06 & .03 & $.27^{*}$ & & \\
\hline
\end{tabular}

Notes: Contrasts were dummy coded, where D1: Self-compassion $=0$, Self-esteem $=1$, Free writing $=0$ and D2: Self-compassion $=0$, Self-esteem $=0$, Free writing $=1$. When analyzing NA as an outcome variable, pre-writing (T2) NA was included as a covariate prior to step 1 .

$\dagger p<.10, * p<.05, * * p<.01$ 


\section{Figures}

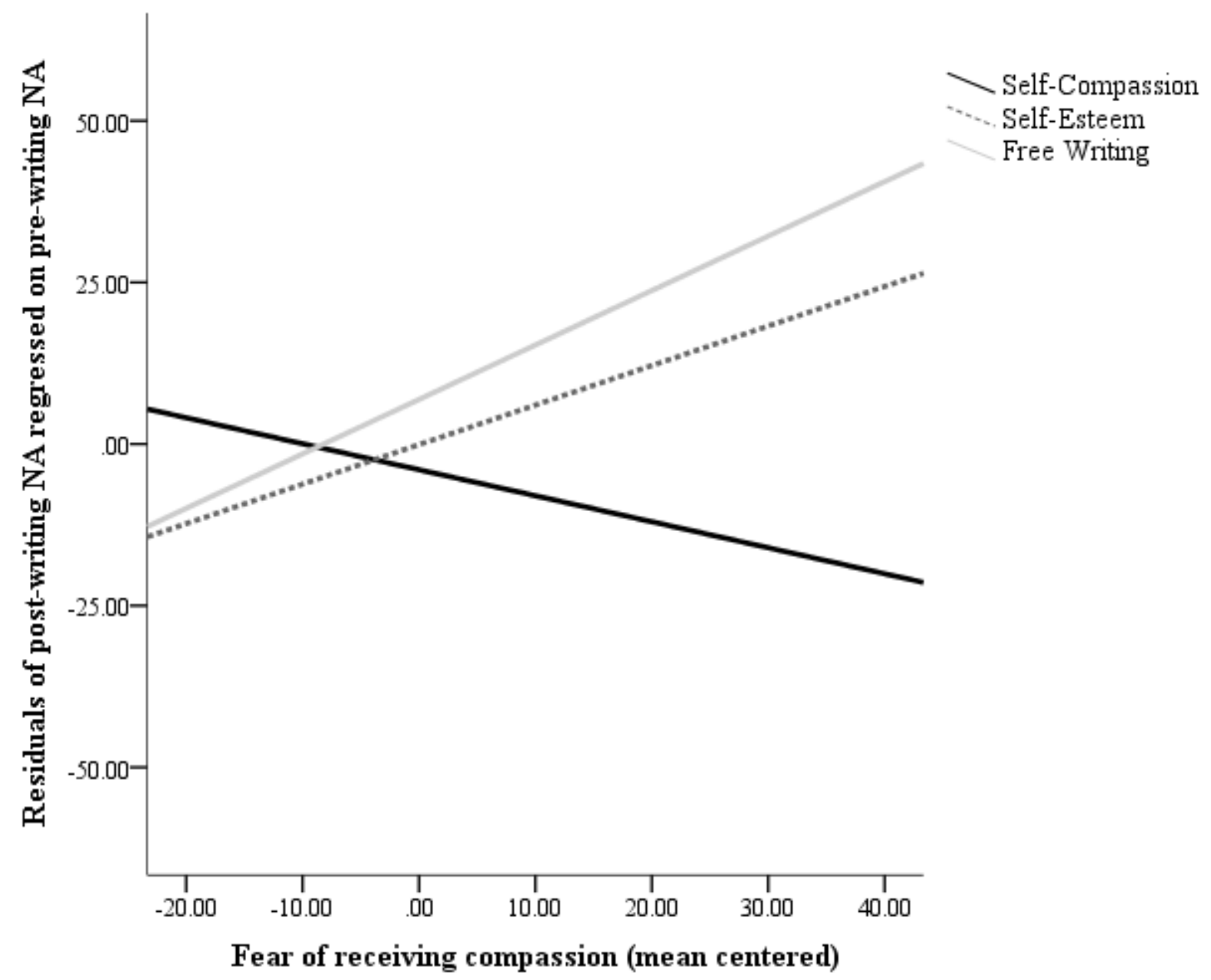

Figure 1. Within-condition simple slope estimates of fear of receiving compassion predicting negative affect post-writing exercise (T3) controlling for pre-writing (T2) negative affect Note: For graphing purposes, pre-writing NA was controlled for by regressing post-writing NA on pre-writing NA and plotting the residuals of the regression (i.e., the difference between participants' post-writing NA and what would be predicted based on their pre-writing NA) 


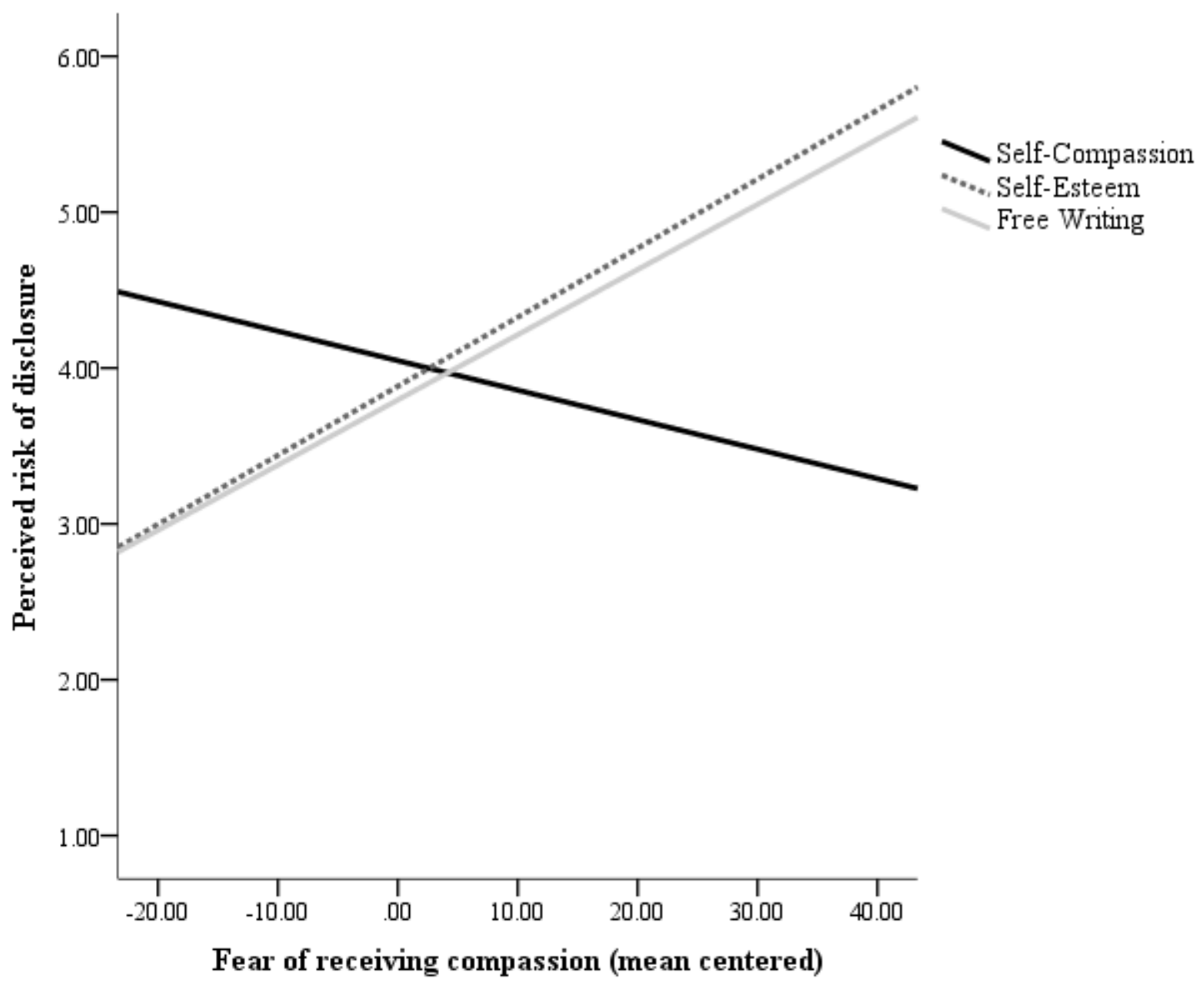

Figure 2. Within-condition simple slope estimates of fear of receiving compassion predicting perceived risk of disclosure 


\section{Supplementary Materials}

\section{Fear of Self-Compassion}

Given that the main intervention of interest in the present study involves a selfcompassion focused writing intervention, and Gilbert et al. (2011) have previously found large correlations between fears of receiving compassion and fears of self-compassion, the relationship between these two constructs is worth some discussion. In the present study, we also administered the fear of self-compassion subscale of the Fears of Compassion Scales, and found a similarly large correlation between it and fear of receiving compassion $(r(83)=.64)$. It is first important to note that while correlated, these two variables shared only $41 \%$ of their variance, meaning they were quite distinguishable types of fears among participants. Hence, participants with heightened fears of receiving compassion from others would have been more likely to fear self-compassion but not to the same degree as their fear of receiving compassion from others. Nevertheless, the question of why self-compassion would be considered a less threatening method to begin exploring the benefits of compassion is worthy of some discussion.

Practically speaking, the act of being self-compassionate remains under one's own control, can be titrated as desired, and does not require self-exposure to others, whereas receiving compassion introduces a greater element of unpredictability to the exercise. Practicing receiving compassion requires a reliance on others to respond with kindness and care, compounding the risk by necessitating trust in others. Furthermore, those who fear self-compassion are often concerned about the possibility of becoming "weak" if they are too kind to themselves or believe they don't deserve to feel better (Gilbert et al., 2011). In the context of the current study where participants understood the goal of such exercises was to alleviate negative feelings, participants who normally fear self-compassion may have chosen to suspend their distaste for being kind to 
themselves as a personal experiment with the goal of alleviating their distress (in other words, using the present study to "test the waters" of self-compassion).

\section{Method}

Procedure. In order to examine the impact of our experimental manipulations on disclosure to others, it was important that the main objective of the study not be known to participants. The description posted online for the research participation pool and information consent letters stated that the study was investigating "strategies for changing perceptions of and feelings toward past negative experiences." All participants completed a general recruitment battery in which they were required to affirm they read and spoke English proficiently, and were proficient at typing in order to be eligible to participate.

For the purposes of the current study, participants were asked to select a negative experience that (a) occurred in the last five years, (b) made them feel badly about themselves at the time of their participation, (c) involved failure, humiliation, rejection, or a combination of these feelings, and (d) they had either not discussed or had discussed very little with anyone previously. Participants were also instructed not to select any experiences that involved criminal activity, abuse or neglect (physical or sexual), or traumatic events as defined by the fifth edition of the Diagnostic and Statistical Manual of Mental Disorders (DSM-5; American Psychiatric Association, 2013). These experiences were excluded to minimize the possible harm caused to participants during the study.

\section{Experimental manipulations.}

\section{Self-compassion condition.}


(1) It's part of life to struggle with adversity, but these experiences are just a normal part of life. In the box below, write down ways in which other people also experience events that are similar to the one you described.

(2) Many times people get carried away with their emotions. In the box below, try to put psychological distance between yourself and your emotions, and write about the event in a detached, objective fashion.

(3) In the box below, write a paragraph expressing kindness, understanding, and concern toward yourself, much like you would write a supportive letter to a friend if this had happened to him or her.

\section{Self-esteem condition.}

(1) In times like these, it is easy to forget our strengths. In the box below, write down your positive characteristics and indications that you are competent and valuable.

(2) When you have a bad experience, you can try to interpret events in a way that makes you feel better about yourself. In the box below, write a paragraph about the experience, explaining how what happened was not your fault.

(3) When we are faced with a past failure, we can remind ourselves of past successes. In the box below, write a paragraph about a time when you were in a similar situation and you did something that made things turn out better.

\section{Writing control condition.}

(1) In the box below, write about your thoughts, really letting yourself go and exploring your deepest thoughts about the negative experience you selected. 
(2) In the box below, write about your emotions, really letting yourself go and exploring your deepest feelings about the negative experience you selected.

(3) In the box below, write about your beliefs, really letting yourself go and exploring your deepest beliefs about the negative experience you selected.

Data analyses. As the Pearson correlation has been found to be an inadequate measure of internal consistency for two-item composites, and Cronbach's alpha tends to underestimate their true reliability, the Spearman-Brown coefficient tends to be the least biased measure of two-item reliability (Eisinga, te Grotenhuis \& Pelzer, 2012). Thus, the Spearman-Brown coefficient was used as a measure of internal consistency for our composite of negative affect.

\section{Data Integrity and Preliminary Analyses}

Missing data for individual items were imputed using the expectation-maximization method for each measure separately. Missing data were not imputed when a participant did not complete the majority of a particular scale. Overall, the percentage of data imputed across measures was less than $.01 \%$ for the Fears of Receiving Compassion scale and the Distress Disclosure Index, and 0\% for all items administered during the experimental session in-lab. Little's (1988) MCAR tests for fears of receiving compassion and the DDI were non-significant $\left(\chi^{2}(12)=5.93, p=.92 ; \chi^{2}(12)=29.19, p=.06\right)$, suggesting the data were missing completely at random. When data are missing completely at random and less than 5\% of data is missing, a single imputation using expectation-maximization provides unbiased parameter estimates while improving power of analyses (Enders, 2001; Scheffer, 2002).

Data were screened for extreme outliers, and two potential univariate outliers (> 3 SDs above or below the mean) were identified. One individual was an outlier on post-writing exercise NA, and one participant was an outlier on negative emotion words contained within their letter. 
As the extreme values in each case were within the plausible range, all data were retained and no changes were made prior to the main analyses. No multivariate outliers were found.

An examination of the studentized residuals revealed a significant positive skew in the proportion of negative affect words contained with participants' letters (skewness $=1.24, S E=$ .26). Thus, a square root transformation was performed to satisfy the assumption of multivariate normality (Howell, 2007). When the same analysis was conducted on the transformed variable, skewness of the residuals was within the normal range ( skewness $=.04$ ).

\section{Equivalence of Groups}

There were no significant differences between participants in the three conditions in mean age $(F(2,78)=.20, p=.82)$, ethnic background $\left(\chi^{2}(20)=24.35, p=.23\right)$, fear of receiving compassion $(F(2,82)=.84, p=.44)$, distress disclosure tendencies $(F(2,82)=2.27, p=.11)$, how badly they felt about their negative experience $(F(2,82)=.62, p=.54)$, or to what degree they had previously disclosed about their negative experience to others $(F(2,82)=.62, p=.54)$. The overall mean for the amount participants had previously disclosed their negative experience was $2.20(S D=1.08)$ out of 5 , suggesting that participants across conditions were selecting experiences they had not fully shared with others previously. Pre-writing exercise (T2), groups did not significantly differ on NA $(F(2,82)=1.13, p=.33)$. See Table 1 for means and standard deviations. 


\section{References for Supplementary Materials}

American Psychiatric Association. (2013). Diagnostic and statistical manual of mental disorders (5th ed.). Washington, DC: Author.

Eisinga, R., te Grotenhuis, M., \& Pelzer, B. (2012). The reliability of a two-item scale: Pearson, Cronbach, or Spearman-Brown? International Journal of Public Health, 58, 637-642.

Enders, C.K. (2001). A primer on maximum likelihood algorithms available for use with missing data. Structural Equation Modeling, 8, 128-141.

Gilbert, P., McEwan, K., Matos, M., \& Rivis, A. (2011). Fears of compassion: Development of three self-report measures. Psychology and Psychotherapy: Theory, Research, and Practice. 84, 239-255.

Howell, D.C. (2007). Statistical methods for psychology (6th ed.). Belmont, CA: Thomson Wadsworth.

Little, R.J. (1988). A test of missing completely at random for multivariate data with missing values. Journal of the American Statistical Association, 83, 1198-1202.

Scheffer, J. (2002). Dealing with missing data. Research Letters in the Information and Mathematical Sciences, 3, 153-160. 


\section{Supplementary Table 1}

Final step of hierarchical linear regressions in which fear of receiving compassion interacted with condition to predict perceived risk of disclosure controlling for perceived severity of the event

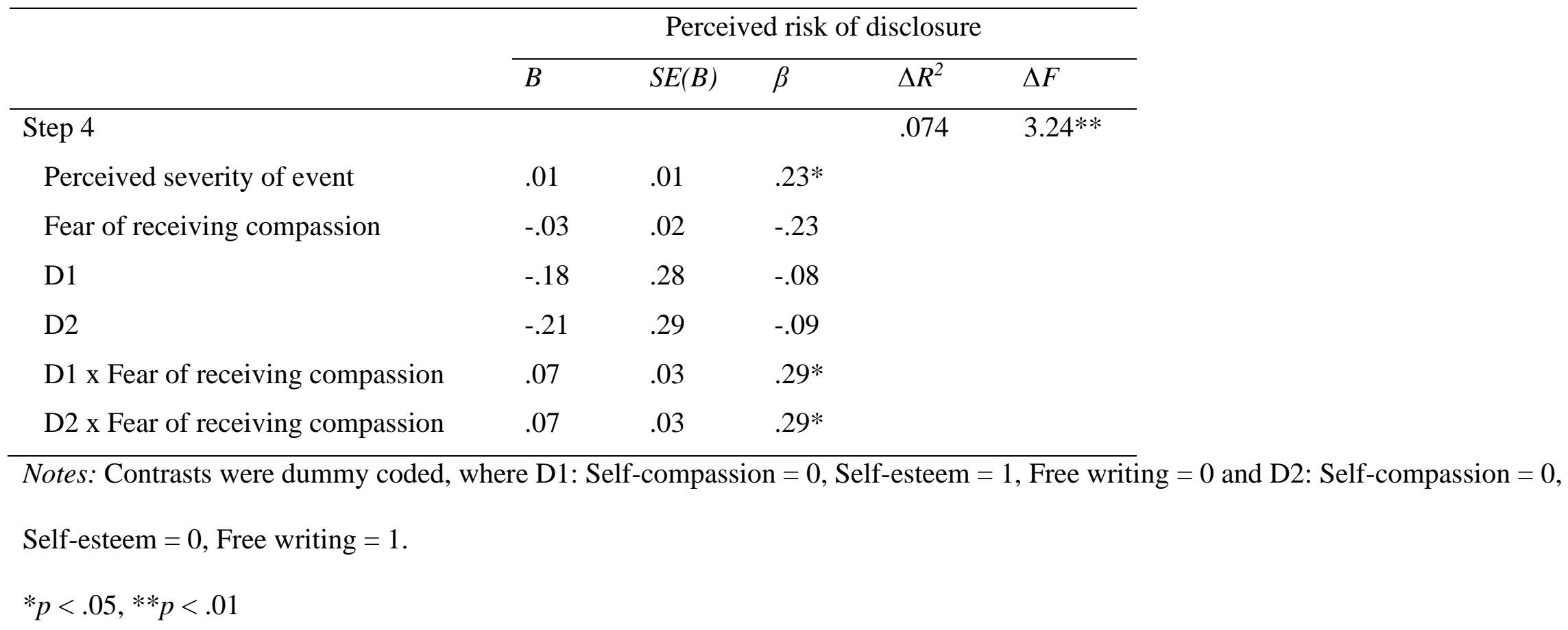

